INPLASY

PROTOCOL

To cite: Heng et al. Safety and efficacy of acupuncture for treatment of cough variant asthma: a systematic review protocol. Inplasy protocol 202110105. doi:

10.37766/inplasy2021.1.0105

Received: 25 January 2021

Published: 28 January 2021

Corresponding author: Heng Zhaorui

753624374@qq.com

Author Affiliation:

BeiJing university of traditional chinese medicine.

Support: 2018-HX-99.

Review Stage at time of this submission: The review has not yet started.

Conflicts of interest: None.

\section{Safety and efficacy of acupuncture for treatment of cough variant asthma: a systematic review protocol}

Heng, Z1; Jing, H²; Xing, W3; Lu, H4; Qing, Z5; Xuan, W6; Tao, L7.

Review question / Objective: We observe safety and efficacy of acupuncture therapy in cough variant asthma.

Condition being studied: Cough variant asthma (CVA) is a special type of asthma, cough is the only or mainly clinical manifestation. There are no obvious symptoms or signs such as wheezing and shortness of breath, but the airway is highly responsive. At present ,the treatment of CVA is mainly included inhale glucocorticoid and bronchodilator with oral corticosteroid and leukotriene regulator.

Information sources: The English databases and the Chinese databases will be searched. The English databases are as following: Web of science, PubMed, Springer, Medline, Cochrane Library, EBase, WHO International Clinical Trials Registry Platform (ICTRP), and the Chinese databases are China National Knowledge Infrastructure Database (CNKI), Wan Fang Database, Chinese Scientific Journal Database (VIP), and Chinese Biomedical Literature Database. we will also search the relevant systematic reviews, reference list of studies and conference abstracts will be searched.Recently completed studies and ongoing studies will be searched.

INPLASY registration number: This protocol was registered with the International Platform of Registered Systematic Review and Meta-Analysis Protocols (INPLASY) on 28 January 2021 and was last updated on 28 January 2021 (registration number INPLASY202110105).

\section{INTRODUCTION}

Review question / Objective: We observe safety and efficacy of acupuncture therapy in cough variant asthma.
Condition being studied: cough variant asthma (CVA) is a special type of asthma, cough is the only or mainly clinical manifestation. There are no obvious symptoms or signs such as wheezing and shortness of breath, but the airway is highly responsive. At present, the treatment of 
CVA is mainly included inhale glucocorticoid and bronchodilator with oral corticosteroid and leukotriene regulator

\section{METHODS}

Search strategy: Data analysis was performed using RevMan5.3.5 software provided by the Cochrane Collaboration. Subgroup analysis will be carried out according to the different types of ATs, characteristics of participants, and outcome measures. A risk ratio (RR) with $95 \%$ confidence intervals (Cls) will be used to estimate the dichotomous outcomes, and the continuous data will be analyzed by mean difference (MD) or standard MD (SMD)with $95 \%$ Cls. We will assess heterogeneity by visually inspecting the forest plots to detect nonoverlapping Cls and by investigating $X 2$ (with $P$ value $>.10$ indicating no heterogeneity) and 12 statistic. $1250 \%$ will be considered as representing substantial heterogeneity and the random effect model is adopt to analyze, while $12<50 \%$ will be taken as evidence of no heterogeneity and the fixed effect model will be used for statistics.

Participant or population: Study participants in different age ranges with a confirmed diagnosis of CVA can be included without restricting nationality, sex, race, occupation and education.Patients with a diagnosis of chronic obstructive pulmonary, cystic fibrosis, or other lung diseases, cardiovascular disease, renal failure, hepatitis, cancer, mental disorder were excluded.

Intervention: The group only treated with acupuncture, such as auricular needle, acusector, fire needle, scalp acupuncture.

Comparator: The group treated with no therapy or placebo acupuncture or sham acupuncture.

Study designs to be included: All relevant randomized controlled trials and cohort studies in English or Chinese on acupuncture for CVA can be included.
Eligibility criteria: Study participants in different age ranges with a confirmed diagnosis of CVA can be included without restricting nationality, sex, race, occupation and education.

Information sources: The English databases and the Chinese databases will be searched. The English databases are as following: Web of science, PubMed, Springer, Medline, Cochrane Library, EBase, WHO International Clinical Trials Registry Platform (ICTRP), and the Chinese databases are China National Knowledge Infrastructure Database (CNKI), Wan Fang Database, Chinese Scientific Journal Database (VIP), and Chinese Biomedical Literature Database. we will also search the relevant systematic reviews, reference list of studies and conference abstracts will be searched.Recently completed studies and ongoing studies will be searched.

Main outcome(s): Symptom score will be assessed as the primary outcome .besides, blood eosinophils, blood IgE, the pulmonary function change, FeNo, and side effects and adverse events will be observed.

Quality assessment / Risk of bias analysis: The risk of bias in each study will be assessed by 2 independent authors (SX and $C L$ ) using the criteria outlined in the Cochrane Handbook for Systematic Reviews of Interventions. If the number of included studies is more than 10 , we will generate funnel plots to detect reporting biases and small-study effects.

Strategy of data synthesis: Data analysis was performed using RevMan5.3.5 software provided by the Cochrane Collaboration. Subgroup analysis will be carried out according to the different types of ATs, characteristics of participants, and outcome measures. A risk ratio (RR) with 95\% confidence intervals (Cls) will be used to estimate the dichotomous outcomes, and the continuous data will be analyzed by mean difference (MD) or standard MD (SMD)with $95 \% \mathrm{CIs}$. We will assess heterogeneity by visually inspecting the forest plots to detect nonoverlapping Cls 
and by investigating $X 2$ (with $P$ value $>.10$ indicating no heterogeneity) and 12 statistic. $1250 \%$ will be considered as representing substantial heterogeneity and the random effect model is adopt to analyze, while $12<50 \%$ will be taken as evidence of no heterogeneity and the fixed effect model will be used for statistics.

Subgroup analysis: Subgroup analysis will be carried out based on different types of ATs, characteristics of participants, and outcome measures.

Sensitivity analysis: We will take sensitivity analyses to test the robustness and reliability of the results, if the dates are sufficient. Sensitivity analysis mainly focuses on research characteristics or types, such as methodological quality, and the effects on total effect are examined by excluding certain lowquality studies or non-blinded studies.

Country(ies) involved: China.

Keywords: cough variant asthma, safety and efficacy, acupuncture.

Contributions of each author:

Author 1 - Heng Zhaorui.

Author 2 - Jing Han.

Author 3 - Xing Wangjian.

Author 4 - Lu Hanze.

Author 5 - Qing Zhang.

Author 6 - Xuan Wanjing.

Author 7 - Tao Linjiang. 